\title{
ISO Standards for Interoperability: a comparison ${ }^{1}$
}

\author{
K. Kosanke \\ CIMOSA Association e.V., Stockholmer Str. 7, \\ D-71034 Böblingen, Germany \\ kosanke@cimosa.de
}

\begin{abstract}
The lack of interoperability between ICT systems is becoming more and more a bottleneck in the collaboration and co-operation of enterprises. Cooperating parties have to exchange business information and have to have the same understanding of the meaning of the exchanged information and to trust both the communication itself and the validity of its contents. The paper reviews two ISO standards, which are aiming on the interoperability issue comparing the different aspects of these standards and introduces a new work item in standardisation, which expects support from two current European initiatives on interoperability and others. The paper is intended to guide further standard developments by identifying current solutions and their relation with one another.
\end{abstract}

\section{Introduction}

Operating in the global business environment requires worldwide co-operations between enterprises sharing their core competencies in order to exploit short-term market opportunities. Besides the timely availability of products and services, the real time exchange of related business information between the co-operating partners is even more important. Such exchanges are needed for both operational control and to an even larger extend for the decision-making processes during the establishment of the cooperation like market opportunity exploration and co-operation planning and implementation. Therefore, both the easy communication between the people involved and the quality of interoperation between the supporting systems of information and communication technology (ICT) play a key role in such co-operations. The urgent need for organisational interoperation and decision support on all levels of the

\footnotetext{
${ }^{1}$ Presented at I-ESA'2005, Geneva, Switzerland, 2005-02-21/25; published in Konstantas et al (Eds.), Interoperability of Enterprise Software and Applications, Springer-Verlag London 2006, ISBN 1-84628-151-2
} 
enterprise operation is recognised in the communities of business and academia as well as in standardisation [1].

Major issues in global collaboration and co-operation of enterprises are the problems in communication between people, between people and ICTs and between different ICTs. Whereas the first one is due to different cultures, languages and even professional jargons and can only be addressed by either a common language, which is very unlikely, or through translations between the different languages and meanings. The other two problem areas originate from the different software and hardware implementations of the information and communication technology. These areas require both human and machine understanding of the exchanged information.

But what is the meaning of interoperability? First of all interoperability is domain specific. Besides the three domains of people, people and ICT, and ICT itself identified above, there are the different business domains like industry, finance, health, each one having sub-domains like categories of humans (managers, experts, operators), of devices (controllers, actuators, sensors), and of systems (computers, machines, communication networks) with their specific needs for interoperation.

But the ability to interoperate within the ICT supported business arena is a rather complex task. The heterogeneity of ICT implementation is such that there exist different solution spaces depending on the combination of existing systems and in many cases such solutions are not transferable to other cases. According to Chen and Doumeingts [2], who reported on the results of a European initiative on a development for road maps to interoperability, there exist several levels of interoperability. They identified interaction between two systems can at least take place at the three levels: data, resource and business process and interoperability may therefore be achieved on multiple levels: inter-enterprise coordination, business process integration, semantic application integration, syntactic application integration, and physical integration.

The recommendations in Chen and Doumeingts are to address the subject of interoperability through three main research themes or research domains: enterprise modelling, ontologies, and architectures and platforms. These three areas deal with a) the representation of the inter-networked organisation to establish interoperability requirements; b) address the semantics necessary to assure interoperability; and c) define the implementation solution to achieve interoperability. General state of the art reports have been issued by both the European Network of Excellence (NoE) initiative INTEROP [3] and the EU Integrated Project (IP) ATHENA [4]. Standardisation in these areas has been and is continuously addressed by both international standards organisations and industry consortia. A multiplicity of standards exists in the three fields identified above.

\section{Definitions on Interoperability}

There exist numerous definitions on interoperability; e.g. a very careful chosen web search produced 22 entries on interoperability. Selected examples are:

Interoperability: 
1. achieved only if the interaction between two systems can, at least, take place at the three levels: data, resource and business process with the semantics defined in a business context (Chen, Doumeingts) [2]

2. ability of two or more systems or components to exchange information and to use the information that has been exchanged (IEEE) [5].

3. ability to communicate with peer systems and access their functionality (Vernadat) [6]

4. ability of different types of computers, networks, operating systems, and applications to work together effectively, without prior communication, in order to exchange information in a useful and meaningful manner. There are three aspects of interoperability: semantic, structural and syntactical (from www) [7]

5. (Computer Science) ability to exchange and use information (usually in a large heterogeneous network made up of several local area networks) (WordNet) [8]

Related definitions are:

1. Interoperable: (computer Science) able to exchange and use information (WordNet) [8]

2. Interoperation: implies that one system performs an operation on behalf of another (Chen, Doumeingts) [2]

3. Interoperation may occur between two (or more) entities that are related to one another in one of three ways (ISO 14258) [9]:

a. Integrated where there is a standard format for all constituent systems

b. Unified where there is a common meta-level structure across constituent models, providing a means for establishing semantic equivalence

c. Federated where models must be dynamically accommodated rather than having a predetermined meta-model

IEC TC 65/290/DC [10] identifies degrees of compatibility depending on the quality of communication and application features (see Figure 1).

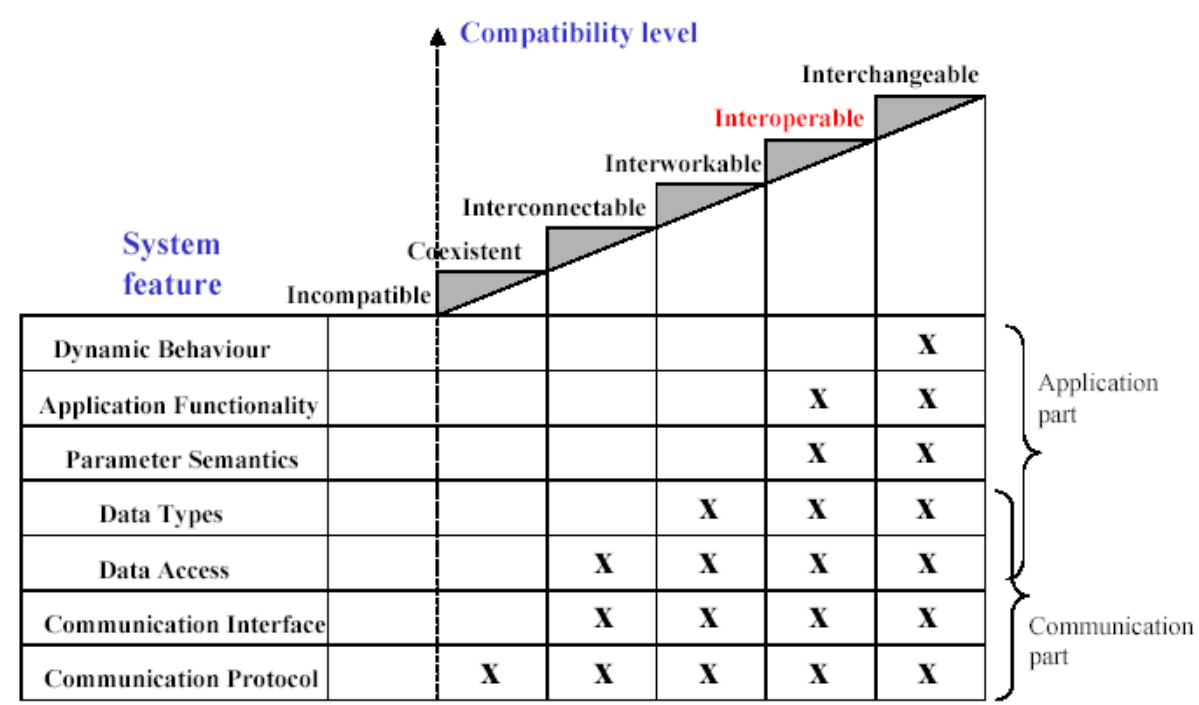


Figure 1 Compatibility levels (adapted from IEC TC 65/290/DC) [10]

The related definitions of the compatibility levels in Figure 1 are:

1. Incompatibility: inability of two or more devices to work together in the same application

2. Coexistence: ability to of two or more devices operate independently of one another in the same communication network

3. Interworkability: ability of two or more devices to support transfer of device parameters

4. Interoperability: ability of two or more devices to work together in one or more applications

5. Interchangeability: ability of two or more devices to replace each other in working together in one or more application

Another attempt to categorise interoperation has been published by Stegwee and Rukanova [11]. Starting from the assumption of communication as the main concept in interoperation and using the IEEE definition of interoperability, the authors have defined a framework, which is shown in Figure 2. The framework identifies three types of communication and enables the identification of relevant standards to support communication between the different components of organisational interoperation. The authors favour the ISO - OSI layer model as a means to support interoperation.

\begin{tabular}{|c|c|c|c|c|}
\hline Type & Purpose & Technical & Human & Process \\
\hline Interconnectivity & $\begin{array}{l}\text { Enables two } \\
\text { systems to } \\
\text { communicate } \\
\text { with each other }\end{array}$ & $\begin{array}{l}\text { Communication } \\
\text { standards, like } \\
\text { TCP/IP or X.25 }\end{array}$ & $\begin{array}{l}\text { Communication } \\
\text { systems like } \\
\text { speech and } \\
\text { writing }\end{array}$ & $\begin{array}{l}\text { Providing for } \\
\text { external inputs and } \\
\text { outputs }\end{array}$ \\
\hline Interchangeability & $\begin{array}{l}\text { Enables two } \\
\text { systems to } \\
\text { exchange } \\
\text { information }\end{array}$ & $\begin{array}{l}\text { Data } \\
\text { representation } \\
\text { standards, like } \\
\text { ASCII or HTML }\end{array}$ & $\begin{array}{l}\text { Language } \\
\text { systems like } \\
\text { natural language } \\
\text { and vocabularies }\end{array}$ & $\begin{array}{l}\text { Displaying the } \\
\text { same behavior in } \\
\text { terms of } \\
\text { input/output }\end{array}$ \\
\hline Interoperability & $\begin{array}{l}\text { Enables two } \\
\text { systems to } \\
\text { operate together } \\
\text { as one }\end{array}$ & $\begin{array}{l}\text { Interaction } \\
\text { standards like } \\
\text { SMTP or SOAP }\end{array}$ & $\begin{array}{l}\text { Behavioral } \\
\text { scenarios and } \\
\text { procedures, } \\
\text { attached to e.g. } \\
\text { military orders }\end{array}$ & $\begin{array}{l}\text { Providing for } \\
\text { external controls } \\
\text { on process } \\
\text { behavior }\end{array}$ \\
\hline
\end{tabular}

Figure 2 Identifying types of interoperation and related of standards (from [11])

The related definitions of the types of communication in Figure 2 are:

1. Interconnectivity: ability to exchange information at a network, syntactical level

2. Interchangeability: ability to use information at a presentation, semantic level

3. Interoperability - ability to use information at an application, pragmatic level. 
The mapping of the definitions of interoperation given in the two frameworks is presented in table 1, which shows the difference in terminology as well as the difference in scope of the term interoperation. Especially the term interchangeablity is used with quite different meaning. Whereas it is used for an intermediate level of communication in [11] it identifies the ultimate interoperation in [10].

Table 1: categories of interoperation

\begin{tabular}{|l|l|}
\hline IEC TC 65/290/DC) [10] & Stegwee and Rukanova [11] \\
\hline & interconnectivity \\
\hline interworkability & interchangeability \\
\hline interoperability & interoperability \\
\hline interchangeability & \\
\hline
\end{tabular}

However, it seems very unlikely that in reality interoperability or interoperation on a larger scale will occur in any one of the three ways identified in ISO 14258 (see above), but in a mixture of those. Assuming a global environment there will be neither possibility for global unification nor for global integration and even federation in the dynamic mode as identified above seems very hard to achieve without any a priory knowledge about the entities that have to interoperate. The two standards analysed in the following try to provide this a-priory knowledge by creating profiles of their entities - applications and manufacturing software units, respectively.

\section{ISO 15745 -Industrial automation systems and integration - Open systems application integration frameworks [12]}

The standard consists of four parts: Part 1: Generic reference description; Part 2: Reference description for ISO 11898-based control systems; Part 3: Reference description for IEC 61158-based control systems; Part 4: Reference description for Ethernet-based control systems. ${ }^{2}$

This standard outlines an Application Integration Framework (AIF) - a set of elements and rules for describing application interoperability profiles, which will enable a common environment for integrating applications and sharing life cycle information in a given application domain. The generic elements and rules are providing for developing templates for Application Interoperability Profiles (AIPs), and their component profiles - process profiles, resource profiles, and information exchange profiles.

Such profiles may describe profiles based upon particular technologies and therefore makes this standard applicable to application integration frameworks developed for industrial automation environments. Environments such as discrete manufacturing, process automation, electronics assembly, semiconductor fabrication, wide-area material handling, and other automation and control sectors such as utility automa-

2 Note: Application here means industrial automation applications, which comprise several types of interconnected devices, which perform a variety of functions. 
tion, agriculture, off-road vehicles, medical and laboratory automation, and public transport systems.

\section{ISO 16100 Industrial automation systems and integration - Manufacturing software capability profiling for interoperability [13]}

The standard consists of four parts as well: Part 1: Framework (relating to ISO 15745); Part 2: Profiling Methodology; Part 3: Interface services, protocols and capability templates; Part 4: Conformance test methods, criteria, and reports.

ISO 16100 specifies a framework for the interoperability of a set of software products used in the manufacturing domain and to facilitate integration into manufacturing applications. The framework addresses models for information exchange, software objects, interfaces, services, protocols, capability profiles, and conformance test methods. The standard specifies a methodology for constructing profiles of manufacturing software capabilities and requirements for interface services and protocols used to access and edit capability profiles and associated templates used in the capability profiling method. In addition, conformance test method and criteria for the capability profiling of a manufacturing software unit are specified.

\section{Relations between the two standards}

The main content identified in each standard is shown in Table 2. It identifies subtle differences owing partly to the difference in scope of the two standards. Whereas Framework elements in ISO 16100 are solely concerned with the interfacing requirements for interoperability of manufacturing software units (MSUs), their roles and the entities they have to support, ISO 15745 identifies a larger set of such elements needed to support interoperability between the components of applications. ${ }^{3}$ Similarly for framework rules where again ISO 15745 provides a larger set. However, some of the missing items in ISO 16100 are listed under framework aspects, an entry that does not exist in ISO 15745.

The latter uses the concepts of life cycle and model to classify the requirements in terms of a set of interfaces, services, components and configurations intended to guide the developers of industrial automation applications.

The three integration model types identified in Table 2 correspond to three different profile classes and a complete integration model of an application corresponds to a set of application interoperability profiles (AIPs), which identify the particular interfaces, services, protocols, and timing used by the different components within the application.

3 Note: Template is seen differently in the two standards. Being an element in 15745 it is identified as a rule in 16100 . 
Table 2: content of ISO 15745 and ISO 16100

\begin{tabular}{|c|c|}
\hline $\begin{array}{l}\text { ISO } 15745 \text { Open systems application } \\
\text { integration frameworks }\end{array}$ & $\begin{array}{l}\text { ISO } 16100 \text { Manufacturing software ca- } \\
\text { pability profiling for interoperability }\end{array}$ \\
\hline $\begin{array}{l}\text { application integration framework (AIF) } \\
\text { framework elements: } \\
\text { diagram, model, profile, template, } \\
\text { specification } \\
\\
\text { - } \\
\text { framework rules: } \\
\text { diagram notation and semantics, } \\
\text { model composition, profile template } \\
\text { and taxonomy, template confor- } \\
\text { mance and exchange syntax }\end{array}$ & $\begin{array}{l}\text { Mfg. software interoperability framework } \\
\text { framework elements: } \\
\text { roles, activities, artefacts associated } \\
\text { with software entities when dealing } \\
\text { with manufacturing process, } \\
\text { information, and resources } \\
\text { framework rules: } \\
\text { relationships, templates, and confor- } \\
\text { mance statements needed for con- } \\
\text { structing a capability class } \\
\text { framework aspects: } \\
\text { - } \quad \text { syntax and semantics shared be- } \\
\text { tween MSUs; } \\
\text { functional relationships exist be- } \\
\text { tween MSUs; } \\
\text { services, interfaces, and proto- } \\
\text { cols offered by MSUs; } \\
\text { ability to provide MSU capabil- } \\
\text { ity profiling. }\end{array}$ \\
\hline \begin{tabular}{|l} 
Concepts and constructs used: \\
- $\quad$ Application life cycle \\
AIF integration models: \\
1.process integration model (PIM) \\
presents views of process con- \\
trol, material and information \\
flow \\
2.resource integration model (RIM) \\
identifies devices, communica- \\
tion networks equipment, mate- \\
rial/product, and operators (hu- \\
mans) needed in the PIM \\
3.information Exchange integration \\
model (IEIM) identifies syntax, \\
semantics, structure, and se- \\
quences of information pro- \\
duced, consumed, organized, \\
stored, and retrieved by the re- \\
sources involved in the PIM
\end{tabular} & $\begin{array}{l}\text { Concepts and constructs used: } \\
-\quad \text { manufacturing software unit (MSU) }\end{array}$ \\
\hline
\end{tabular}


Table 2: content of ISO 15745 and ISO 16100 (continued)

\begin{tabular}{|c|c|}
\hline $\begin{array}{l}\text { ISO } 15745 \text { Open systems application } \\
\text { integration frameworks }\end{array}$ & $\begin{array}{l}\text { ISO } 16100 \text { Manufacturing software ca- } \\
\text { pability profiling for interoperability }\end{array}$ \\
\hline $\begin{array}{l}\text { Application interoperability profiles } \\
\text { (AIPs) consists of: } \\
-\quad \text { one process profile } \\
\text { - } \quad \text { one or more resource profile(s) } \\
\text { one or more information exchange } \\
\text { profile(s) } \\
\text { AIP concepts and rules: } \\
\text { - } \text { combine the interface specification } \\
\text { option selections as required by the } \\
\text { application } \\
\text { shall be a single specification aimed } \\
\text { at providing a defined function } \\
\text { shall comprise a specific combina- } \\
\text { tion of profiles } \\
\text { AIPs shall be constructed by: } \\
\text { 1. documenting the functional re- } \\
\text { quirements as noted by a PIM; } \\
\text { 2. } \text { selecting the appropriate base speci- } \\
\text { fications for the object interfaces } \\
\text { denoted in the integration models; } \\
\text { 3. Selecting (conforming) sets of op- } \\
\text { tions, or subsets, in the base specifi- } \\
\text { cations; } \\
\text { 4. } \\
\text { combining references to a set of } \\
\text { compatible base specifications in } \\
\text { order to meet the identified applica- } \\
\text { tion functional requirement. } \\
\text { 5. describing it in terms of an interface } \\
\text { definition language }\end{array}$ & $\begin{array}{l}\text { Software capability profile } \\
-\quad \text { taxonomy } \\
\text { - } \quad \text { capability classes and rules: } \\
\text { types of mfg. domain, activity, com- } \\
\text { puting system, services, protocols, } \\
\text { supplier, others } \\
\text { - } \quad \text { capability templates and rules } \\
\text { - } \quad \text { common part contains general } \\
\text { information about SMU } \\
\text { - } \quad \text { specific part contains SMU spe- } \\
\text { cific lists of attributes, methods, } \\
\text { resources, constraints, others }\end{array}$ \\
\hline
\end{tabular}

ISO 16100 provides only one concept and construct: the manufacturing software unit (MSU). Again to support interoperability a software capability profile is defined. Capability classes and rules as well as templates and rules provide the elements for constructing the capability profiles of the software units.

The actual process of profile creation is shown as Application Interface Profiles (AIP) construction (ISO 15745) and capability profiling process (ISO 16100) in the lower part of the Table 2. The number of steps identified in the two standards varies from 3 for ISO 16100 to 5 for ISO 15745 . However, the process itself is rather similar considering the difference in scope of the two standards. 


\section{Summary and Conclusions}

The two standards presented in this paper address the issue of interoperability between ICTs. With their focus on interoperability within manufacturing applications and between manufacturing software units, respectively, the two standards are both using the concept of profiles to capture the information needed to identify the capabilities of the entities, which have to interoperate.

In respect to the three types of interoperation identified in ISO 14258 [9] the two standards contribute to a semi federated approach of interoperation at the ICT level, providing means for identifying a priory information that can be mapped or matched at run time to/with the profile of the partner entity.

However, the comparison between the two standards also shows the need for standards harmonisation. Both terminology and structure of the two standards differ and any potential user, who has to employ both standards, will be confused by their difference. Certainly a more thorough analysis of the state of the art in standardisation and an adoption of already established structures, rules and terminologies would reduce such differences as identified for the two standards.

In addition, the two standards do not yet address sufficiently the human aspects of interoperation, which have been identified in Stegwee and Rukanova [11]. For the communication between people and between people and machines, information about the internal structure and the dynamics of the application may be even more important than the information about the potential exchange itself. Business process models can provide such information with their focus on semantic unification and orientation on end-user needs.

Work on standards for interoperability has been started in ISO. In TC 184 SC5/WG1 a new work item 'Requirements for establishing information interoperability in manufacturing-enterprise-processes and their models' will address this aspect of interoperability. New standards have to improve the ICT interoperability as well by providing a base for unifying the needed information exchange between the parties involved, may it be between operational processes during enterprise operation or between their models during decision support. Inputs to this work will also be provided besides others by the two European initiatives ATHENA and INTEROP.

Standardisation for enterprise interoperability is considered an important subject. However, the current state of standardisation is not yet sufficient to allow easy implementation at the operational level. Many of the standards are still on the conceptional level and more details are still required to make them truly useable in the operation. Work is required in areas of languages and supporting platforms, especially for the business process model creation and execution. To enable cross-organisational decision support especially the subject of 'common' semantics has to be addressed. In ISO/CEN 19440 [14] modelling constructs are defined using a meta-model and accompanying text (sufficient to define an intuitive semantics as well as to define a model repository database). However, the capture of finer details of these meanings requires even more detailed formal semantics. Ontologies will play an important role in the area of semantic unification. 


\section{References}

1. Kosanke, K. (1997), Enterprise Integration and Standardisation, in Kosanke, K. \& Nell, J.G. (Eds.). Enterprise Engineering and Integration: Building International Consensus Proceedings of ICEIMT'97 (Intern. Conference on Enterprise Integration and Modelling Technology), Springer-Verlag, pp 613-623

2. Chen, Doumeingts, (2003), European initiatives to develop interoperability of enterprise applications_-basic concepts, framework and roadmap, Annual Reviews in Control 27, pp 153-162

3. INTEROP (2004), Knowledge map of research in interoperability in the INTEROP NoE, Deliverable D1.1, EU-NoE Project IST-508 011, www.interop.noe.org

4. ATHENA, (2004), Document Deliverable D.A1.1.1, Project A1: Enterprise Modelling in the Context of Collaborative Enterprises ATHENA, EU IP- Project - No 507849

5. IEEE (1990), IEEE (Institute of Electrical and Electronics Engineers): Standard Computer Dictionary- A Compilation of IEEE Standard Computer Glossaries

6. Vernadat, F.B. (1996) Enterprise Modelling and Integration: principles and applications; Chapman \& Hall, ISBN 0412605503

7. www (2001) library.csun.edu/mwoodley/dublincoreglossary.html

8. WordNet Browser 1.7.1, Princeton University Cognitive Science Lab

9. ISO 14258 (1999), Industrial automation systems and integration - Concepts and rules for enterprise models, ISO TC 184 SC5

10. IEC TC 65/290/DC (2002), Device Profile Guideline, TC65: Industrial Process Measurement and Control

11. Stegwee, R.A., Rukanova, B.D. (2003). Identification of Different Types of Standards for Domain-Specific Interoperability. In Proceedings of the Workshop on Standard Making: A Critical Research Frontier for Information Systems, John L. King and Kalle Lyytinen, (eds.), Seattle, WA, December 12-14, 2003, pp. 161- 170. available on line at: http://www.si.umich.edu/misq-stds/proceedings/139_161-170.pdf Standard Making: A Critical Research Frontier for Information Systems, MISQ Special Issue Workshop

12. ISO 15745 (2000), Industrial automation systems and integration - Open systems application integration frameworks, ISO/TC/184/SC5

13. ISO 16100 (2002), Industrial automation systems and integration - Manufacturing software capability profiling for interoperability, ISO/TC/184/SC5

14. CEN-ISO 19440 (2003), Constructs for Enterprise Modelling CEN TC 310/WG1 and ISO TC 184/SC5/WG1 